\title{
Efeitos ambientais sobre características pré-desmama em bovinos da Raça Nelore Mocha ${ }^{1}$
}

\author{
Environmental effects on preweaning performance traits in \\ Nellore beef cattle
}

\author{
Ana Maria do Val Santiago Pereira ${ }^{2}$; Carolina Amália de Souza Dantas Muniz ${ }^{3 *}$
}

\section{Resumo}

O objetivo deste trabalho foi analisar os dados de pesagens de animais da raça Nelore Mocha, provenientes de um rebanho no Estado de São Paulo no período de 2007 a 2010, avaliando a influência de fatores ambientais sobre as características pré-desmama observadas. Foram utilizadas 205 observações de peso à desmama ajustado para 205 dias de idade (P205), ganho médio diário do nascimento à desmama (GMD) e dias para o animal ganhar $160 \mathrm{~kg}$ do nascimento à desmama (D160) pelo Método dos Quadrados Mínimos, utilizando-se um modelo contendo os efeitos fixos de grupo de contemporâneo (data de nascimento, mês de nascimento, sexo e data juliana de desmama), pai e idade da vaca ao parto e, como efeito aleatório, o erro. Utilizando o mesmo modelo, foram analisadas 124 observações de peso ajustado aos 90 dias de idade (P90). Os efeitos de grupo de contemporâneo (GC) e de pai influenciaram significativamente todas as características estudadas. Para P90 verificou-se o efeito da idade da vaca ao parto $(\mathrm{P}<0,05)$. As médias e desvios padrão observados foram de $140,67 \pm 20,08 \mathrm{~kg}$, $0,539 \pm 0,097 \mathrm{~kg} /$ dia e 306,42 $\pm 55,84$ dias, para P205, GMD e D160, respectivamente. Os animais mais leves foram os nascidos em fevereiro $(115,65 \mathrm{~kg})$ e os mais pesados em julho $(156,18 \mathrm{~kg})$. Os machos apresentaram valores de médias estimadas para GC de 5,8\% superiores que as fêmeas. A importância dos efeitos não genéticos evidencia a necessidade destes serem considerados no modelo de análise, visto que influenciaram as características estudadas.

Palavras-chave: Creep-feeding, D160, efeito materno, estação de monta

\begin{abstract}
The objective of this study was to analyze a data set with 205 records from a Nellore breed cattle, evaluating the influence of environmental factors on weaning weight adjusted to 205 days of age (W205), preweaning average daily gain (ADG) and number of days to gain $160 \mathrm{~kg}$ (D160). The data came from a farm in São Paulo State and were collected from 2007 to 2010. The statistical analysis was accomplished using the least squares method, with a model that included the fixed effects of contemporary group, sire and cow age at calving, and error as random effect. The same model was used to analyze 124 records of weight adjusted to 90 days of age (W90). The effects of contemporary group and sire were significant. The cow age at calving was not significant for any performance trait analyzed, except for W90 $(\mathrm{P}<0.05)$. The estimated means were $140.67 \pm 20.08 \mathrm{~kg}, 0.539 \pm 0.097 \mathrm{~kg}$ /day and 306.42 \pm 55.84 days, for W205, ADG e D160, respectively. The weightless calves were born in February $(115.65 \mathrm{~kg})$ and the heaviest in July $(156.18 \mathrm{~kg})$. The males were 5.8\% superior than females. The importance of non genetic effects indicates the need to consider them when estimating genetic parameters and breeding values for selection purpose.
\end{abstract}

Key words: Creep-feeding, D160, maternal effect, mating season

\footnotetext{
${ }^{1}$ Trabalho de conclusão de curso da primeira autora como parte das exigências para obtenção do grau de Médica Veterinária no Centro das Faculdades Metropolitanas Unidas, FMU, SP.

${ }^{2}$ Médica Veterinária, Fazenda Macaúba, Boa Esperança do Sul, SP. E-mail: amdoval@uol.com.br

${ }^{3}$ Prof $^{\mathrm{a}} \mathrm{Dr}^{\mathrm{a}}$ do Dept $^{\mathrm{o}}$ de Zootecnia, Universidade Estadual de Londrina, UEL, Londrina, PR. E-mail: muniz@uel.br

* Autor para correspondência
} 


\section{Introdução}

A bovinocultura de corte é caracterizada, basicamente, por sistemas de produção constituídos, em sua maioria, pelas fases de cria, recria e terminação. A fase de cria compreende todos os processos de produção envolvidos desde a escolha e cuidado dos progenitores até o desmame do produto (MUNIZ et al., 2005). Fries (1997), relatou que cerca de $2 / 3$ dos custos de produção de carne bovina estão associados à fase de cria, compreendendo desde a recria de fêmeas de reposição até os processos de gestação, lactação e desmame. As características da pré-desmama são de grande importância econômica em bovinos de corte. Nesta fase, obtêm-se os primeiros dados sobre o desempenho do animal, além de se poder avaliar a capacidade criadeira da mãe. Ou seja, as características da pré-desmama podem ser utilizadas para avaliar tanto o potencial genético de crescimento do bezerro como a habilidade materna da vaca.

O desenvolvimento do bezerro até a desmama pode ser medido pelo peso à desmama corrigido para a idade do animal, pelo ganho médio diário do nascimento à desmama e, ainda, pelo número de dias que um bezerro necessita para ganhar $160 \mathrm{~kg}$ do nascimento à desmama.

A seleção para peso éimportante na bovinocultura de corte e sua utilização em larga escala, quando bem conduzida, pode trazer benefícios econômicos. No entanto, critérios de seleção que incluem o peso em determinada idade, buscando animais com altos valores para peso, muitas vezes não são desejados. Isto porque, a seleção apenas para peso, em longo prazo, pode levar, por resposta correlacionada, a maior peso adulto e, conseqüentemente, a maiores exigências de mantença, difíceis de serem atendidas nos sistemas de produção em pastagens. Assim, incrementos genéticos, sejam em ganho de peso ou em pesos, resultam em aumento de consumo de alimento e em aumento dos custos de produção (ALBUQUERQUE; FRIES, 1998). Fries e Albuquerque (1998) afirmaram que a tradução mais usual de precocidade é velocidade de crescimento e pode ter sua medida no ganho médio diário (GMD) ou dias para ganhar $160 \mathrm{~kg}$ (D160).

Vários são os fatores que influenciam o crescimento do animal na fase pré-desmama. Dentre eles, há os inerentes ao próprio indivíduo, como a raça, o sexo e a individualidade, e os relacionados ao ambiente e manejo, como a habilidade materna, nível nutricional, ano e estação de nascimento, idade da mãe ao parto e idade do próprio bezerro, entre outros (BOCCHI; TEIXEIRA; ALBUQUERQUE, 2004).

Desta forma, os efeitos de ambiente que têm sido considerados importantes na fase de desmama são idade da vaca, sexo e idade do bezerro, além do grupo de contemporâneos, que engloba os efeitos de rebanho, ano, estação e grupo de manejo (CARDOSO; CARDELINO; CAMPOS, 2001).

O objetivo deste trabalho foi verificar os principais efeitos não genéticos que influenciaram o peso a desmama ajustado para 205 dias de idade (P205), ganho médio diário do nascimento à desmama (GMD) e dias para o animal ganhar 160 $\mathrm{kg}$ (D160) em animais da raça Nelore Mocha.

\section{Material e Métodos}

Os dados utilizados no presente estudo são referentes a 205 dados de pesagens de bovinos da raça Nelore Mocha, provenientes do Controle de Desenvolvimento Ponderal (CDP) de animais registrados pela Associação Brasileira dos Criadores de Zebu (ABCZ), coletada no período de 2007 a 2010 na Fazenda Macaúba, situada no município de Boa Esperança do Sul, Estado de São Paulo. A fazenda está localizada a $21^{\circ} 53^{\prime} 22,1^{\prime \prime}$ de latitude sul e $48^{\circ} 32^{\prime} 41,6^{\prime \prime}$ de latitude leste. Caracteriza-se por duas estações climáticas distintas, uma estação seca, no período de abril a setembro, e o período das águas, de outubro a março, no qual as chuvas são abundantes. 
A vegetação nativa é de cerrado, sendo que grande parte da fazenda encontra-se em solo arenoso. As áreas de pastagens são formadas por capim Brachiaria, sendo cerca de $70 \% \mathrm{~B}$. humidicola e $30 \%$ B. brizantha. Os animais foram criados, exclusivamente, em regime de pasto, onde também receberam suplementação mineral. Os bezerros tiveram acesso ao creep-feeding durante todo o período de aleitamento.

Tanto a prática da inseminação artificial como a monta natural foram usadas nos acasalamentos e existe uma concentração de acasalamentos nos meses de setembro à março.

Para comparar o desempenho dos animais, as observações foram analisadas pelo Método dos Quadrados Mínimos Generalizados (SAS, 2001), pelo seguinte modelo fixo: $\mathrm{Yijkl}=\mu+\mathrm{GCi}+\mathrm{Pj}+\mathrm{Ik}$ + eijkl, em que Yijkl = observação (desempenho) do 1-ésimo animal, doi-ésimo grupo de contemporâneos; filho do j-ésimo pai, filho da vaca na k-ésima idade ao parto; $\mu=$ média geral; $\mathrm{GCi}=$ efeito fixo do i-ésimo grupo de contemporâneos $(\mathrm{i}=1, \ldots, 24) ; \mathrm{Pj}$ = efeito fixo do $\mathrm{j}$-ésimo pai $(\mathrm{j}=1, \ldots, 11)$; $\mathrm{Ik}=$ efeito fixo da k-ésima idade da vaca ao parto, em anos ( $\mathrm{k}$ $=3, \ldots, 8)$; eijkl = erro aleatório associado a cada observação ijkl, suposto normalmente distribuído com média zero e variância $\sigma^{2}$. Utilizando modelo semelhante, foram analisadas 124 observações de peso ajustado aos 90 dias de idade (P90).

A partir das médias estimadas para os grupos de contemporâneos, foram calculadas as médias para sexo do bezerro. Foram também calculadas as médias para ano de nascimento, mês de nascimento, e data juliana de desmama, as quais foram comparadas pelo teste Duncan $(\mathrm{P}<0,05)$. A variável grupo de contemporâneos (GC), considerou os efeitos de ano de nascimento, mês de nascimento, sexo do animal e data juliana de desmama do animal. Grupos de contemporâneos contendo menos que cinco animais $(\mathrm{GC}<5)$ foram eliminados das análises.

\section{Resultados e Discussão}

A média geral estimada para P205 neste estudo foi de 140,67 $\pm 20,08 \mathrm{~kg}$. Souza et al. (2004) observaram média de $160 \mathrm{~kg}$ em animais raça Nelore Mocha, no Estado de São Paulo. No mesmo Estado, Ribeiro et al. (2009) estimaram média de 198,77 kg em uma fazenda de animais da raça Nelore, criados exclusivamente em pastagem com suplementação mineral, à vontade. Santos et al. (2011), obtiveram média de 165,34 kg para a raça Nelore.

A média geral estimada para GMD $(0,539 \pm$ $0,097 \mathrm{~kg} / \mathrm{dia})$ foi próxima à verificada por Cubas et al. (2001), de $0,510 \mathrm{~kg} / \mathrm{dia}$ e por Sarmento et al. (2003), de 0,575 kg/dia, ambos em bovinos da raça Nelore. Verificou-se ainda que os bezerros em média, apresentaram $306 \pm 55,84$ dias para atingir $160 \mathrm{~kg}$ do nascimento à desmama. Souza et al. (2008) estimaram média de D160 de 250 dias, para a raça Nelore. Valor semelhante (245 dias, em média) foi obtido por Garnero et al. (2001). Malhado et al. (2008) observaram média de 271 dias para a característica D160, em animais da raça Nelore. Santos et al. (2011), verificaram 336, 79 dias para a raça Nelore em um rebanho na região Norte do Brasil, para os bezerros ganharem $160 \mathrm{~kg}$ do nascimento ao desmame e Gusmão, Malhado e Martins Filho (2009), verificaram 272, 3 dias para a mesma característica em um rebanho na Bahia.

O resumo das análises de variância das características estudadas é apresentado na Tabela 1. Verifica-se que o efeito de grupo de contemporâneos influenciou significativamente as características estudadas $(\mathrm{P}<0,0001)$, indicando a necessidade de se considerar essa fonte da variação ao comparar-se o desempenho dos animais. O efeito de pai também foi fonte de variação importante $(\mathrm{P}<0,01)$, havendo diferenças distintas entre as progênies dos touros, o que provavelmente indica variabilidade de origem genética. 
Tabela 1. Graus de liberdade (GL) e quadrados médios das fontes de variação incluídas no Modelo para peso ajustado à desmama (P205), ganho médio diário do nascimento à desmama (GMD) e dias para o animal ganhar $160 \mathrm{~kg}$ (D160).

\begin{tabular}{lcccc}
\hline \multicolumn{1}{c}{ Fonte de Variação } & \multirow{2}{*}{ GL } & \multicolumn{3}{c}{ Quadrados Médios } \\
\cline { 3 - 5 } & 23 & $1612,696^{* * * *}$ & $0,037^{* * * *}$ & $10924,127^{* * * *}$ \\
\hline Grupo de Contemporâneos & 10 & $492,332^{* *}$ & $0,012^{* *}$ & $4239,982^{* *}$ \\
Pai & 5 & 84,807 & 0,002 & 47,922 \\
Idade da Vaca & 166 & 200,219 & 0,005 & 1634,944 \\
Resíduo & - & 59,59 & 59,01 & 57,33 \\
\hline $\mathrm{R}^{2}(\%)$ & & & &
\end{tabular}

$* *=\mathrm{P}<0,01 ; * * * *=\mathrm{P}<0,0001$

$\mathrm{R}^{2}=$ coeficiente de determinação

Fonte: Elaboração dos autores.

Geralmente, a idade da vaca apresenta efeito significativo sobre características pré-desmama, em grande parte, devido às alterações na capacidade de produção de leite no decorrer de sua vida útil. No entanto, o efeito de idade da vaca ao parto não foi significativo para nenhuma das características analisadas. Isto pode ser explicado pelo fato da propriedade adotar o sistema de creep-feeding no manejo alimentar dos bezerros. Em termos de seleção de vacas superiores, tal prática dificulta a determinação da habilidade materna. Bezerros desenvolvidos à custa do creep-feeding tendem a mascarar o potencial de produção de leite da mãe e, conseqüentemente, seu valor genético no rebanho (CHIZZOTTI; CHIZZOTTI, 2010).

As médias de P205, GMD e D160, para cada ano de nascimento, foram calculadas a partir das médias estimadas pelo modelo para grupo de contemporâneos (GC) e são apresentadas na Tabela 2. Pode-se verificar que não houve diferença significativa no desempenho dos animais nascidos nos diferentes anos. Entretanto, pode-se observar que os animais nascidos em 2007 e 2008 apresentarem maiores valores para peso à desmama, com maiores valores para ganho de peso e menores valores para dias para atingir $160 \mathrm{~kg}$ do nascimento à desmama. Nos anos seguintes, verifica-se tendência de diminuição dos pesos e ganhos de peso e aumento nos dias para o animal atingir $160 \mathrm{~kg}$. Os bezerros nascidos em 2010 apresentaram menores valores para pesos e menores valores para ganho de peso até a desmama. No entanto, deve-se destacar que poucos dados foram analisados neste ano, com os nascimentos concentrados somente no mês de janeiro, época menos favorável ao desenvolvimento pré-desmama e, além disso, essa diferença não foi significativa (Tabela 2).

A diminuição dos pesos e ganho médio diário ao longo dos anos, bem como aumento nos dias para o animal atingir $160 \mathrm{~kg}$ pode ocorrer principalmente, em razão das variações anuais na disponibilidade das pastagens, seja em função das alterações climáticas, do manejo, adubação e conservação do pasto. Embora, as diferenças observadas no desempenho dos animais, no presente estudo (Tabela 2), não tenham sido significativas, pode ter ocorrido degradação de pastagens ou o manejo inadequado das mesmas no período estudado, refletindo no desempenho dos animais. A maior utilização de sêmen de determinado touro, bem como touro em monta natural, podem também ser responsáveis pelos valores verificados nos pesos entre os anos de nascimento.

As médias de P205, GMD e D160 para os meses de nascimento, conforme as médias estimadas para GC são apresentadas na Tabela 3. Para todas as características analisadas, o período de nascimento mais favorável foi de julho a setembro, concordando com os trabalhos de Bocchi, Teixeira e Albuquerque (2004), Martins et al. (2000), Cubas et al. (2001) e Santos et al. (2011). 
Tabela 2. Médias dos pesos ajustados à desmama (P205), ganhos médios diários do nascimento à desmama (GMD) e dias para o animal ganhar $160 \mathrm{~kg}$ (D160), conforme o ano de nascimento.

\begin{tabular}{ccccc}
\hline \multirow{2}{*}{ Ano de Nascimento } & \multirow{2}{*}{$\mathrm{N}$} & \multicolumn{3}{c}{ Médias } \\
\cline { 3 - 5 } & & P205 $(\mathrm{kg})$ & GMD $(\mathrm{kg} / \mathrm{dia})$ & D160 (dias) \\
\hline 2007 & 59 & $145,08^{\mathrm{a}}$ & $0,561^{\mathrm{a}}$ & $305,21^{\mathrm{a}}$ \\
2008 & 58 & $143,76^{\mathrm{a}}$ & $0,554^{\mathrm{a}}$ & $303,94^{\mathrm{a}}$ \\
2009 & 71 & $133,68^{\mathrm{a}}$ & $0,505^{\mathrm{a}}$ & $329,84^{\mathrm{a}}$ \\
2010 & 17 & $126,40^{\mathrm{a}}$ & $0,463^{\mathrm{a}}$ & $347,03^{\mathrm{a}}$ \\
\hline
\end{tabular}

$\mathrm{N}=$ número de observações

Médias com letras diferentes entre anos têm diferença significativa $(\mathrm{P}<0,05)$.

Fonte: Elaboração dos autores.

Tabela 3. Médias dos pesos ajustados à desmama (P205), ganhos médios diários do nascimento à desmama (GMD) e dias para o animal ganhar $160 \mathrm{~kg}$ (D160), conforme o mês de nascimento.

\begin{tabular}{ccccc}
\hline $\begin{array}{c}\text { Mês de } \\
\text { Nascimento }\end{array}$ & $\mathrm{N}$ & \multicolumn{3}{c}{ Média } \\
\cline { 3 - 5 } Janeiro & 23 & $\mathrm{P} 205(\mathrm{~kg})$ & GMD $(\mathrm{kg} / \mathrm{dia})$ & $\mathrm{D} 160($ dias$)$ \\
Fevereiro & 12 & $128,58^{\mathrm{bc}}$ & $0,482^{\mathrm{bc}}$ & $347,03^{\mathrm{abc}}$ \\
Julho & 18 & $114,98^{\mathrm{c}}$ & $0,414^{\mathrm{c}}$ & $386,48^{\mathrm{a}}$ \\
Agosto & 18 & $160,16^{\mathrm{a}}$ & $0,633^{\mathrm{a}}$ & $275,51^{\mathrm{d}}$ \\
Setembro & 24 & $151,60^{\mathrm{a}}$ & $0,593^{\mathrm{a}}$ & $280,71^{\mathrm{cd}}$ \\
Outubro & 24 & $149,48^{\mathrm{a}}$ & $0,581^{\mathrm{a}}$ & $291,99^{\mathrm{cd}}$ \\
Novembro & 60 & $141,67^{\mathrm{ab}}$ & $0,544^{\mathrm{ab}}$ & $306,38^{\mathrm{bcd}}$ \\
Dezembro & 26 & $141,25^{\mathrm{ab}}$ & $0,542^{\mathrm{ab}}$ & $304,96^{\mathrm{bcd}}$ \\
\hline
\end{tabular}

$\mathrm{N}=$ número de observações

Médias com letras diferentes entre meses têm diferença significativa $(\mathrm{P}<0,05)$.

Fonte: Elaboração dos autores.

Por ser uma época de poucas chuvas, tornase mais favorável à criação dos bezerros, em razão da menor incidência de doenças. Além disso, os bezerros nascidos nesse período também foram favorecidos pela melhor disponibilidade e qualidade de forragem nos meses subseqüentes, principalmente na época em que são desmamados.

Entretanto, bezerros nascidos nos meses de dezembro a fevereiro apresentaram desempenho inferior no período pré-desmama. Os bezerros nascidos nessa época foram desmamados em outubro (entre os dias 283 e 301 do calendário juliano), passando os últimos meses da amamentação, assim como suas mães, na época seca do ano, quando os pastos não dispõem de boa quantidade e qualidade de forragem. Como pode ser verificado na Tabela
4, de acordo com a data juliana de desmama, os bezerros desmamados nessa época apresentaram desempenho pré-desmama inferior aos desmamados em abril (entre os dias 117 e 120 do calendário juliano). Desta forma, o efeito de mês de nascimento sugere a melhor época (julho a setembro) para concentrar os nascimentos dos bezerros, por meio do uso de estação de monta definida. O efeito de mês de nascimento também pode exercer influência nos desempenhos apresentados em cada ano. Conforme verificado, os anos de 2007 e 2008 apresentaram alguns GC contendo nascimentos em épocas mais favoráveis para o desenvolvimento pré-desmama do bezerro (julho a setembro). Já em 2009, somente um dos GC apresentou nascimentos no mês de setembro, sendo que o restante dos nascimentos ocorreu nos meses de fevereiro, outubro e novembro. 
Tabela 4. Médias dos pesos ajustados à desmama (P205), ganhos médios diários do nascimento à desmama (GMD) e dias para o animal ganhar $160 \mathrm{~kg}$ (D160), conforme a data juliana de desmama.

\begin{tabular}{ccccc}
\hline \multirow{2}{*}{$\begin{array}{c}\text { Data Juliana de Desmama } \\
\text { (dias) }\end{array}$} & $\mathrm{N}$ & \multicolumn{3}{c}{ Médias } \\
\cline { 3 - 5 } & 22 & $156,60^{\mathrm{a}}$ & GMD $(\mathrm{kg} / \mathrm{dia})$ & D160 (dias) \\
\hline 117 & 30 & $152,34^{\mathrm{a}}$ & $0,616^{\mathrm{a}}$ & $274,85^{\mathrm{d}}$ \\
120 & 10 & $139,87^{\mathrm{ab}}$ & $0,597^{\mathrm{a}}$ & $286,52^{\mathrm{cd}}$ \\
195 & 82 & $152,34^{\mathrm{a}}$ & $0,535^{\mathrm{ab}}$ & $311,14^{\mathrm{cd}}$ \\
286 & 17 & $126,40^{\mathrm{b}}$ & $0,596^{\mathrm{a}}$ & $273,31^{\mathrm{d}}$ \\
293 & 13 & $128,16^{\mathrm{b}}$ & $0,470^{\mathrm{b}}$ & $347,03^{\mathrm{b}}$ \\
294 & 12 & $123,16^{\mathrm{b}}$ & $0,483^{\mathrm{b}}$ & $337,49^{\mathrm{abc}}$ \\
301 & 19 & $120,10^{\mathrm{b}}$ & $0,450^{\mathrm{b}}$ & $369,54^{\mathrm{b}}$ \\
\hline
\end{tabular}

$\mathrm{N}=$ número de observações

Médias com letras diferentes entre datas julianas têm diferença significativa $(\mathrm{P}<0,05)$.

Fonte: Elaboração dos autores.

As médias das características P205, GMD e D160, foram iguais a $142,95 \mathrm{~kg}$ e $134,61 \mathrm{~kg}$, $0,54 \mathrm{~kg} / \mathrm{dia}$ e $0,515 \mathrm{~kg} / \mathrm{dia}, 312,91$ dias e 319,69 dias para machos $(\mathrm{N}=116)$ e fềmeas $(\mathrm{N}=89)$ respectivamente. Verifica-se que os GC contendo machos apresentaram maiores valores para as médias estimadas e a diferença a favor dos machos foi de $8,3 \mathrm{~kg}(5,8 \%)$ para P205 e 0,031 kg/dia $(5,7 \%)$ para GMD. Para D160, a superioridade dos valores estimados para GC contendo machos em relação às fêmeas foi de apenas 2,2\%.

Na Tabela 5 pode-se verificar o resumo da análise de variância para P90. A média estimada para P90 foi $87,46 \mathrm{~kg}$, a idade da vaca ao parto foi importante fonte de variação para esta característica $(\mathrm{P}<0,05)$. Nos primeiros meses de vida os bezerros ainda dependem muito do aleitamento materno, assim quando foi considerada uma idade inferior a idade padrão de 205 dias o efeito da idade da mãe apresentou-se significativo. Aos 205 dias, possivelmente, devido à prática de suplementação dos bezerros com a utilização de creep-feeding, bezerros filhos de vacas mais velhas ou mais novas não foram penalizados pela menor produção de leite de suas mães. Provavelmente, esses bezerros compensaram o menor consumo de leite com a suplementação no creep-feeding.

Tabela 5. Graus de liberdade e quadrados médios das fontes de variação incluídas no Modelo para P90.

\begin{tabular}{lcc}
\hline \multicolumn{1}{c}{ Fonte de Variação } & Graus de Liberdade & Quadrados Médios (P90) \\
\hline Grupo de Contemporâneos & 15 & $161,678^{* * *}$ \\
Pai & 9 & $179,471^{* * *}$ \\
Idade da Vaca ao Parto & 5 & $161,412^{* *}$ \\
Resíduo & 94 & 61,631 \\
$\mathrm{R}^{2}(\%)$ & - & 63,66 \\
\hline
\end{tabular}

$* * \mathrm{P}<0,05 ; * * * \mathrm{P}<0,005 ; \mathrm{R}^{2}=$ coeficiente de determinação

Fonte: Elaboração dos autores. 
A influência da idade da vaca sobre o desempenho até a desmama tem sido reportada por diversos autores (BOCCHI; TEIXEIRA; ALBUQUERQUE, 2004; TEIXEIRA; ALBUQUERQUE, 2003; SARMENTO et al., 2003; BIEGELMEYER; MENEZES; CAMPOS, 2007; SANTOS et al., 2011).

\section{Conclusões}

Os resultados obtidos neste trabalho permitem concluir que:

A inclusão dos fatores ambientais no modelo de análise das características de desempenho produtivo na fase pré-desmama é necessária para que avaliações genéticas sejam adequadamente ajustadas e, conseqüentemente, mais precisas e confiáveis.

A utilização do creep-feeding, possivelmente afetou o efeito da idade da mãe sobre o desempenho do bezerro no período pré-desmame.

Recomenda-se a utilização da estação de monta, visto que bezerros que nasceram de Julho a Setembro apresentaram melhor desempenho pré-desmama.

\section{Referências}

ALBUQUERQUE, L. G.; FRIES, L. A. Selection for reducing ages of marketing units in beef cattle. In: WORLD CONGRESS ON GENETICS APPLIED TO LIVESTOCK PRODUCTION. Proceedings... Armindale: WCGALP, 1998, v. 27, p. 235-238.

BIEGELMEYER, P.; MENEZES, L. M.; CAMPOS, L. T. Efeito da idade da vaca sobre o peso ao nascimento e peso à desmama de bovinos da raça Angus. In: CONGRESSO DE INICIAÇÃO CIENTÍFICA, 16; ENCONTRO DE PÓS GRADUAÇÃO DA UNIVERSIDADE FEDERAL DE PELOTAS, 9., 2007, Pelotas. Anais... Universidade Federal de Pelotas: [s.n.], 2007.

BOCCHI, A. L.; TEIXEIRA, R. A.; ALBUQUERQUE, L. G. Idade da vaca e mês de nascimento sobre o peso ao desmame de bezerros nelore nas diferentes regiões brasileiras. Acta Scientiarum, Maringá, v. 26, n. 4, p. 475-482, 2004.
CARDOSO, F. F.; CARDELlINO, R. A.; CAMPOS, L. T. Fatores ambientais que afetam o desempenho de nascimento à desmama de bezerros Angus criados no Rio Grande do Sul. Revista Brasileira de Zootecnia, Viçosa, MG, v. 30, n. 2, p. 326-335, 2001.

CHIZZOTTI, M. L.; CHIZZOTTI, F. H. M. Bovinocultura de corte - módulo II. Apostila acadêmica. Universidade Federal de Lavras: Lavras, MG, 2010. Disponível em: $<$ www.dzo.ufla.br/mario/files/apostilaGZO118_2010> Acesso em: 15 nov. 2010.

CUBAS, A. C.; PEROTTO, D.; ABRAHÃO, J. J. S.; MELLAS, S. C. Desempenho até a desmama de bezerros Nelore e cruzas com Nelore. Revista Brasileira de Zootecnia, Viçosa, MG, v. 30, n. 3, p. 694-701, 2001.

FRIES, L. A. Cruzamentos em gado de corte. In: SIMPÓSIO SOBRE PECUÁRIA DE CORTE, 4., 1997, Pirracicaba. Anais... Piracicaba: FEALQ, 1997. p. 109128.

FRIES, L. A.; ALBUQUERQUE, L. G. Pressuposições e restrições dos modelos animais com efeitos maternos em gado de corte. In: COSTA, M. J.; CROMBERG, V. U. (Ed.). Comportamento materno em mamíferos: bases teóricas e aplicações aos ruminantes domésticos. Jaboticabal: SBEt-ETCO, 1998. p. 179-214.

GARNERO, A. D. V.; LOBO, R. B.; BEZERRA, L. A. F.; OLIVEIRA, H. N. Comparação entre alguns critérios de seleção para crescimento na Raça Nelore. Revista Brasileira de Zootecnia, Viçosa, MG, v. 30, n. 3, p. 714718, 2001.

GUSMÃO, F. B.; MALHADO, C. H. M.; MARTINS FILHO, P. L. S. C. Tendências genéticas, fenotípicas e ambientais para D160 e D240 em bovinos Nelore no estado da Bahia. Revista Ciência Agronômica, Forlaleza, v. 40, n. 2, p. 301-305 abr.jun. 2009.

MALHADO, C. H. M.; CARNEIRO, P. L. S.; MARTINS FILHO, R.; AZEVÊDO, D. M. M. R.; AFFONSO, P. R. A. M.; SOUZA, J. C. correlações genéticas entre características de crescimento e parâmetros da curva em bovinos da Raça Nelore. Revista Científica de Produção Animal, Teresina, v. 10, n. 2, 2008.

MARTINS, G. A.; MARTINS FILHO, R.; LIMA, F. A. M.; LÔBO, R. N. B. Influência de fatores genéticos e de meio sobre o crescimento de bovinos da raça Nelore no Estado do Maranhão. Revista Brasileira de Zootecnia, Viçosa, MG, v. 29, n. 1, p. 103-107, 2000.

MUNIZ, C. A. S. D.; CARVALHEIRO, R.; FRIES, L. A.; QUEIROZ, S. A. Dois critérios de seleção na prédesmama de bovinos da raça Gir. 1. Estimativas de parâmetros genéticos. Revista Brasileira de Zootecnia, Viçosa, MG, v. 34, n. 3, p. 807-815, 2005. 
RIBEIRO, S.; ELER, J. P.; BALIEIRO, J. C. C.; FERRAZ, J. B. S.; PEDROSA, V. B.; MATTOS, E. C. Influência da interação genótipo $\mathrm{x}$ ambiente sobre $\mathrm{o}$ peso à desmama em bovinos da raça Nelore. Arquivo Brasileiro de Medicina Veterinária e Zootecnia, Belo Horizonte, v. 61, n. 3, p. 668-675, 2009.

SANTOS, G. C. J.; LIRA, T. S.; PEREIRA, L. S.; LOPES, F. B.; FERREIRA, J. L. Efeitos não genéticos sobre características produtivas em rebanhos Nelore criados na região Norte do Brasil. Acta Veterinária, Mossoró, v. 5, n. 4, p. 358-392, 2011.

SARMENTO, J. L. R.; PIMENTA FILHO, E. C.; RIBEIRO, M. N.; MARTINS FILHO, R. Efeitos ambientais e genéticos sobre ganho em peso diário de bovinos Nelore no estado da Paraíba. Revista Brasileira de Zootecnia, Viçosa, MG, v. 32, n. 2, p. 325-330, 2003.
SOUZA, J. C.; CAMPOS DA SILVA, L. O.; SIMÕES, G. H.; MOSER, J. T.; OSTAPECHEN, J.; PINTO, P. H. N.; RUVIERO, V.; MALHADO, C. H. M.; FERRAZ FILHO, P. B.; FREITAS, J. A.; SERENO, J. R. B. Tendências ambientais e genéticas para características produtivas de bovinos da raça Nelore. Archivos Latinoamericanos de Producción Animal, Mayaguez, Porto Rico, v. 16, n. 2, p. 84-89, 2008.

SOUZA, M. C. A.; FERRAZ FILHO, P. B.; SILVA, L. O. C.; SOUZA, J. C.; MALHADO, C. H. M. Efeitos genéticos e ambientais sobre pesos à desmama de bovinos da raça Nelore Mocha, na região pecuária Oeste São Paulo - Paraná. Archives of Veterinary Science, Curitiba, v. 9, n. 2, p. 113-118, 2004.

STATISTICAL ANALYSIS SYSTEM - SAS. Statistical analysis system user's guide. Version 8.2. Cary: SAS Institute, 2001. $943 \mathrm{p}$.

TEIXEIRA, R. A.; ALBUQUERQUE, L. G. Efeitos ambientais que afetam o ganho de peso pré-desmama em animais Angus, Hereford, Nelore e mestiços Angus-Nelore e Hereford-Nelore. Revista Brasileira de Zootecnia, Viçosa, MG, v. 32, n. 4, p. 887-890, 2003. 